\title{
DIRECT PRODUCTS AND THE CONTRAVARIANT HOM-FUNCTOR
}

\author{
SIMION BREAZ
}

\begin{abstract}
We prove in ZFC that if $G$ is a (right) $R$-module such that the groups $\operatorname{Hom}_{R}\left(\prod_{i \in I} G_{i}, G\right)$ and $\prod_{i \in I} \operatorname{Hom}_{R}\left(G_{i}, G\right)$ are naturally isomorphic for all families of $R$-modules $\left(G_{i}\right)_{i \in I}$ then $G=0$. The result is valid even we restrict to families such that $G_{i} \cong G$ for all $i \in I$.
\end{abstract}

\section{INTRODUCTION}

It is well known that if $\mathcal{C}$ is a category and $G$ is an object in $\mathcal{C}$ then the homfunctors $\operatorname{Hom}_{\mathcal{C}}(-, G)$ and $\operatorname{Hom}_{\mathcal{C}}(G,-)$, from $\mathcal{C}$ into the category of sets, are very useful tools. For instance, the universal property of a direct product can be described by the fact that all covariant hom-functors $\operatorname{Hom}_{\mathcal{C}}(G,-)$ preserve direct products, [6, pp.70 and 117]: for any $G$ and any family $\mathcal{F}=\left(G_{i}\right)_{i \in I}$ of objects in $\mathcal{C}$ such that there exists the (direct) product $\prod_{i \in I} G_{i}$, there is natural bijection

$$
\Phi_{\mathcal{F}}: \operatorname{Hom}_{\mathcal{C}}\left(G, \prod_{i \in I} G_{i}\right) \rightarrow \prod_{i \in I} \operatorname{Hom}_{\mathcal{C}}\left(G, G_{i}\right) .
$$

If $\mathcal{C}$ has a null object (e.g. $\mathcal{C}$ is the category of pointed sets, of groups, of pointed spaces or a category of modules) there are also canonical maps $u_{i}: G_{i} \rightarrow \prod_{i \in I} G_{i}$ for all $i \in I$. The family $\bar{u}_{i}=\operatorname{Hom}_{R}\left(u_{i}, G\right), i \in I$, induces a natural homomorphism

$$
\Psi_{\mathcal{F}}: \operatorname{Hom}_{\mathcal{C}}\left(\prod_{i \in I} G_{i}, G\right) \rightarrow \prod_{i \in I} \operatorname{Hom}_{\mathcal{C}}\left(G_{i}, G\right) .
$$

It is easy to see that in general $\Psi_{\mathcal{F}}$ is not a bijection (e.g. for pointed sets or for vector spaces), and it is a natural question whether we can add conditions on $G$ such that the induced maps $\Psi_{\mathcal{F}}$ are isomorphisms for all families $\mathcal{F}$.

In the following we will prove (in ZFC) that for module categories the only condition we can put is the trivial one, $G=0$. This result is valid even if we restrict to all families $\mathcal{F}$ which consists in modules which are isomorphic copies of $G$. A similar theorem was proved for abelian groups by Goldsmith and Kolman in [5. Theorem 3.3] under an additional set theoretic hypothesis (there exists a strongly compact cardinal), and our result provides an answer to the questions from the end of [5]: Does ZFC suffice to prove that for every non-zero abelian group $G$ there is a family $\left(G_{i}\right)_{i \in I}$ of abelian groups (eventually with $G_{i} \cong G$ for all $i \in I$ ) such that $\operatorname{Hom}\left(\prod_{i \in I} G_{i}, G\right)$ and $\prod_{i \in I} \operatorname{Hom}\left(G_{i}, G\right)$ are not isomorphic?

The answer presented here is satisfactory since other commuting properties of hom-functors are studied only in similar settings. For instance the main characterizations for (self-)small modules (see [1, Proposition 1.1]), using ascending

1991 Mathematics Subject Classification. 16D90, 18A23, 18A30, 08A10, 08B25, 20K30.

Key words and phrases. direct product, hom-functor, pure-injective module. 
chains of submodules, are given for the hypothesis that the natural homomorphism $\bigoplus_{i \in I} \operatorname{Hom}_{R}\left(G, G_{i}\right) \rightarrow \operatorname{Hom}_{R}\left(G, \bigoplus_{i \in I} G_{i}\right)$ is an isomorphism, while slender modules are characterized in a similar way in [2, Corollary III.1.5].

\section{Modules, $G$, such that $\operatorname{Hom}(-, G)$ Preserves direct products}

Let $R$ be a unital ring, $G$ an $R$-module and $\mathcal{F}=\left(G_{i}\right)_{i \in I}$ a family of modules. As before, we denote by $u_{i}: G_{i} \rightarrow \prod_{i \in I} G_{i}$ the canonical injections and by

$$
\Psi_{\mathcal{F}}: \operatorname{Hom}_{R}\left(\prod_{i \in I} G_{i}, G\right) \rightarrow \prod_{i \in I} \operatorname{Hom}_{R}\left(G_{i}, G\right)
$$

the natural homomorphism induced by the family $\bar{u}_{i}=\operatorname{Hom}_{R}\left(u_{i}, G\right), i \in I$. Following the terminology used in [5], we say that $G$ is naturally cosmall if $\Psi_{\mathcal{F}}$ is an isomorphism for all families $\mathcal{F}$. If $\Psi_{\mathcal{F}}$ is an isomorphism for all families $\mathcal{F}$ which consist of isomorphic copies of $G$, then $G$ is called naturally self-cosmall. We will prove that the only naturally self-cosmall module (hence the only naturally cosmall module) is the trivial module 0 . In order to do this we start with a technical lemma.

Lemma 1. Let $G$ be an $R$-module. If $\mathcal{F}=\left(G_{i}\right)_{i \in I}$ is a family of $R$-modules, $\varphi: \bigoplus_{i \in I} G_{i} \rightarrow \prod_{i \in I} G_{i}$ is the natural homomorphism and

$$
\Upsilon_{\mathcal{F}}: \operatorname{Hom}_{R}\left(\bigoplus_{i \in I} G_{i}, G\right) \rightarrow \prod_{i \in I} \operatorname{Hom}_{R}\left(G_{i}, G\right)
$$

is the natural isomorphism induced by the canonical injections $v_{i}: G_{i} \rightarrow \bigoplus_{i \in I} G_{i}$, then

$$
\Psi_{\mathcal{F}}=\Upsilon_{\mathcal{F}} \operatorname{Hom}_{R}(\varphi, G) .
$$

Proof. Let $\pi_{i}: \prod_{i \in I} \operatorname{Hom}_{R}\left(G_{i}, G\right) \rightarrow \operatorname{Hom}_{R}\left(G_{i}, G\right)$ be the canonical projections. The standard proof, [3. Theorem 43.1], of the isomorphism $\operatorname{Hom}_{R}\left(\bigoplus_{i \in I} G_{i}, G\right) \cong$ $\prod_{i \in I} \operatorname{Hom}_{R}\left(G_{i}, G\right)$ shows that $\operatorname{Hom}_{R}\left(v_{i}, G\right)=\pi_{i} \Upsilon_{\mathcal{F}}$ for all $i \in I$.

Similarly, $\operatorname{Hom}\left(u_{i}, G\right)=\pi_{i} \Psi_{\mathcal{F}}$. Now $\varphi v_{i}=u_{i}$, so that, $\operatorname{Hom}\left(v_{i}, G\right) \operatorname{Hom}(\varphi, G)=$ $\operatorname{Hom}\left(u_{i}, G\right)$, that is, $\pi_{i} \Upsilon_{\mathcal{F}} \operatorname{Hom}(\varphi, G)=\pi_{i} \Psi_{\mathcal{F}}$ for all $i \in I$. The conclusion follows immediately.

Theorem 2. The following are equivalent for a module $G$ :

(1) $G$ is naturally cosmall;

(2) $G$ is a naturally self-cosmall module;

(3) $G=0$.

Proof. Only $(2) \Rightarrow(3)$ requires a proof. Let $G$ be a naturally self-cosmall module. For every cardinal $\lambda$ we consider the canonical exact sequence

$$
0 \rightarrow G^{(\lambda)} \stackrel{\varphi}{\rightarrow} G^{\lambda} \rightarrow G^{\lambda} / G^{(\lambda)} \rightarrow 0
$$

Applying the previous lemma, it follows that $\operatorname{Hom}_{R}(\varphi, G)$ is an isomorphism, hence the exactness of these sequences are preserved if we apply the functor $\operatorname{Hom}_{R}(-, G)$. Then $G$ is pure-injective as a consequence of [4, 1.2.19(e)].

Moreover, $\operatorname{Hom}_{R}\left(G^{\lambda} / G^{(\lambda)}, G\right)=0$ for all $\lambda$ and we will prove that this is possible only if $G=0$.

Fix $\lambda$ an infinite ordinal. We observe that the set $H=\left\{(g)_{\kappa<\lambda} \mid g \in G\right\}$ of constant functions

$$
\lambda=\{\kappa \mid \kappa<\lambda\} \rightarrow G, \kappa \mapsto g
$$


is a submodule in $G^{\lambda}$, and we claim that $\left(H+G^{(\lambda)}\right) / G^{(\lambda)}$ is pure in $G^{\lambda} / G^{(\lambda)}$. In order to prove this claim we consider a system of equations

$$
\text { (S) } \sum_{j=1}^{k} a_{i j} X^{j}=\left(g^{i}\right)_{\kappa<\lambda}+G^{(\lambda)}, i=1, \ldots, n, a_{i j} \in R,\left(g^{i}\right)_{\kappa<\lambda} \in H, n, k \in \mathbb{N},
$$

in $G^{\lambda} / G^{(\lambda)}$. Suppose that the tuple

$$
\left(\bar{x}^{1}=\left(x_{\kappa}^{1}\right)_{\kappa<\lambda}+G^{(\lambda)}, \ldots, \bar{x}^{k}=\left(x_{\kappa}^{k}\right)_{\kappa<\lambda}+G^{(\lambda)}\right)
$$

represents a solution for $(\mathcal{S})$ in $G^{\lambda} / G^{(\lambda)}$. Then for every $i=1, \ldots, n$ the equalities

$$
\sum_{j=1}^{k} a_{i j} x_{\kappa}^{j}=g^{i}
$$

hold for almost all $\kappa<\lambda$. It follows that there is $\nu<\lambda$ such that the constant functions

$$
y^{1}=\left(x_{\nu}^{1}\right)_{\kappa<\lambda}, \ldots, y^{k}=\left(x_{\nu}^{k}\right)_{\kappa<\lambda} \in G^{\lambda}
$$

satisfy the equalities $\sum_{j=1}^{k} a_{i j} y^{j}=\left(g^{i}\right)_{\kappa<\lambda}$ for all $i=1, \ldots, n$. Then the tuple

$$
\left(y^{1}+G^{(\lambda)}, \ldots, y^{k}+G^{(\lambda)}\right)
$$

represents a solution for $\mathcal{S}$ in $\left(H+G^{(\lambda)}\right) / G^{(\lambda)}$.

Therefore $\left(H+G^{(\lambda)}\right) / G^{(\lambda)} \cong G$ is pure in $G^{\lambda} / G^{(\lambda)}$. But $G$ is pure-injective, hence $\left(H+G^{(\lambda)}\right) / G^{(\lambda)}$ is a direct summand in $G^{\lambda} / G^{(\lambda)}$.

If we suppose $G \neq 0$ we obtain $\operatorname{Hom}_{R}\left(G^{\lambda} / G^{(\lambda)}, G\right) \neq 0$, a contradiction.

Acknowledgement. I would like to thank to Ciprian Modoi and Phill Schultz for illuminating discussions on subjects related to the main result of this note. I am thankful to the referee for her/his suggestions who helped me to improve the presentation of the paper.

\section{REFERENCES}

[1] D. M. Arnold and C. E. Murley: Abelian groups, $A$, such that $\operatorname{Hom}(A,-)$ preserves direct sums of copies of A, Pacific J. Math., 56, (1975), 7-21.

[2] P. Eklof, A. Mekler: Almost Free Modules: Set-theoretic methods, Revised edition , North-Holland Mathematical Library vol. 65 (2002).

[3] L. Fuchs: Infinite Abelian Groups, Vol I, Academic Press (1970).

[4] R. Göbel, J. Trlifaj: Endomorphism Algebras and Approximations of Modules, Expositions in Mathematics 41, Walter de Gruyter Verlag, Berlin (2006).

[5] B. Goldsmith and O. Kolman: On cosmall Abelian groups, J. Algebra, 317, (2007), $510-518$.

[6] S. MacLane: Categories for the working mathematician, Graduate texts in mathematics 5, Springer-Verlag, 1998.

Babeş-Bolyai University, Faculty of Mathematics and Computer Science, Str. Mihail Kogălniceanu 1, 400084 Cluj-Napoca, Romania

E-mail address: bodo@math.ubbcluj.ro 